\title{
Deconvolution of microarray data predicts transcriptionally regulated protein kinases of Plasmodium falciparum
}

\author{
Wei Zhao ${ }^{1,2}$, Justin Dauwels ${ }^{1,2}$, Jacquin Niles ${ }^{1,3}$, and Jianshu Cao ${ }^{1,4}$ \\ ${ }^{1}$ Singapore-MIT Alliance for Research and Technology, Singapore; ${ }^{2}$ School of Electrical \\ and Electronic Engineering, Nanyang Technological University; ${ }^{3}$ Biological Engineering Department, \\ Massachusetts Institute of Technology; and ${ }^{4}$ Department of Chemistry, Massachusetts Institute of Technology \\ wzhao3@e.ntu.edu.sg; jdauwels@ntu.edu.sg; \{jcniles,jianshu\}@mit.edu
}

\begin{abstract}
We have developed a computational approach which predicts the protein kinases that may regulate the transition between the blood developmental stages of Plasmodium falciparum (P. falciparum). To improve the accuracy of our prediction, synchronized gene expression levels are reconstructed from the observed microarray data generated by the ensembles of non-synchronized cells. Peaks in annotated protein kinase transcript levels are hypothesized to directly correlate with the period when the encoded protein kinases function temporally. Therefore, protein kinases, which putatively regulate a given developmental stage transition, are identified by their peak in synchronized gene expression levels. By analyzing publicly available microarray data set, a few protein kinases are considered to be strongly associated with developmental stage transition. Two of these (PF13_0211, PFB0815w) have recently been implicated in the schizont to ring transition [1], [2]. Another one of these identified (MAL7P1.144) has been found to influence erythrocyte membrane in both trophozoite and schizont [3]. Overall, these results suggest that further functional analysis of the other protein kinases we have predicted may reveal new insights into $P$. falciparum blood stage development.
\end{abstract}

Keywords-Plasmodium falciparum, protein kinase, and gene expression level.

\section{BACKGROUND}

Malaria is a mosquito-borne disease of humans, affecting about forty percent of the global population and leading to over one million deaths annually [4]. This disease is caused by any of four species of Plasmodium parasites, of which $P$. falciparum is the most virulent one and is responsible for the majority of human fatalities.

$P$. falciparum is transmitted by mosquito. When an infected mosquito bites a person, the $P$. falciparum is injected into the bloodstream in the form of sporozoites. Once it invades the human body, $P$. falciparum sequentially infects the liver and blood. In particular, sporozoites first travel to the human liver and multiply in the liver cells. After several days, tens of thousands of $P$. falciparum in the form of merozoites exit from infected liver cells and begin to invade red blood cells (RBCs). This invasion is also called the intra-erytrhocytic developmental cycle (IDC). During IDC, $P$. falciparum invades and propagates rapidly in $\mathrm{RBCs}$, causing the common symptoms of malaria such as chills, fever and sweating.

To understand the parasite process in human blood, microarray technology is being used to measure the gene expression level of $P$. falciparum in IDC. It has been reported that all of the three stages of infected RBCs (iRBCs) in IDC-rings, trophozoite and schizont-have an apparent gene expression pattern, with specific subsets of gene displaying consistent behavior in the given stage [5], [6]. However, the key proteins which control the transition between stages have not been identified. It is not surprising given that those proteins can be the potential targets in the treatment of malaria. In a recent study, it is found that the protein kinase PF13_0211 regulates the parasite egress in the schizont stage [1]. Consequently we hypothesize that there may exist other protein kinases which also play key roles in regulating the stage transition in IDC.

Hundreds of protein kinases have been identified in $P$. falciparum [7], [8]. Considering the difficulty to infer the function of each protein kinase from biological experiments, we propose a computational approach to predict the protein kinases which may regulate the stage transitions in IDC. Hence further experimental study can focus on the few protein kinases predicted by our approach, rather than on hundreds of them. The proposed approach is inspired by two observations. Firstly, the peak in the gene expression level usually indicates that the function of the encoded protein is required [9]. Therefore, the protein kinases which appear a high expression level when stage transition occurs have a high likelihood to be involved in regulating this transition. Secondly, owing to the lack of synchrony among iRBCs, the peak pattern just mentioned is not readily apparent from the observed microarray data, which is measured from an ensemble of non-synchronized iRBCs. In the microarray experiment, for example, iRBCs are usually synchronized by sorbitol treatments in the beginning. However, due to the individual diversity, iRBCs gradually lose synchrony over time as the experiment continues [5], [10]. Now it is still an open problem how to ensure the synchrony of iRBCs in microarray experiment. 
We analyze the microarray experiment and identify three main factors which drive the iRBCs out of synchronization: diversity of infection time, diversity of growth rate, and newly infected RBCs. Dan et al. have presented a gene expression deconvolution algorithm to remove the effects of diversity in cell growth rate [11]. However, growth rate is not the only reason leading to decaying synchrony. Therefore we model the decaying synchrony and proposed our method to reconstruct the synchronized gene expression levels from observed microarray data. Then the desired protein kinases can be better predicted from the synchronized gene expression levels.

The materials section describes the microarray data used in this work. The details of proposed approach are presented in the methods section. Our results are discussed in the results section. The conclusions are given in the end of this paper.

\section{MATERIALS}

A publicly available microarray data for the $P$. falciparum strains 3D7 is used in this work [5], [10]. The expression levels of 4022 oligonucleotide sequences has been previously measured at fifty three time points with one hour interval. As some data are not valid in the original data set, only the oligonucleotide sequences with valid data over all fifty three time points are used in this work. After the quality filtering, 2623 oligonucleotide sequences are collected in total.

Eighty-two protein kinases involved in the $P$. falciparum life cycle are identified by retrieving the PlasmoDB database. Since protein kinases may have several unique oligonucleotide sequences, eighty-two of protein kinases were represented by one hundred and seven of the oligonucleotide sequences. For the protein kinases which have multiple expression curves, average trace is calculated from multiple curves to represent the protein. Finally, the gene expression levels of eighty-two protein kinases are collected from public data set and used in this study.

\section{Methods}

There are two main components. Firstly, a method is proposed to extract synchronized gene expression levels from the microarray data: the decaying synchrony among iRBCs are modeled as a series of cell age distributions changing radically over time. The process of how gene expression curve are generated from non-synchronized cells is modeled as a linear system. The synchronized gene expression curves are reconstructed by solving the discrete linear system as a discrete linear inverse problem. Secondly, based on the synchronized gene expression curves, protein kinases are prioritized by their likelihood of being involved in the desired stage transition.

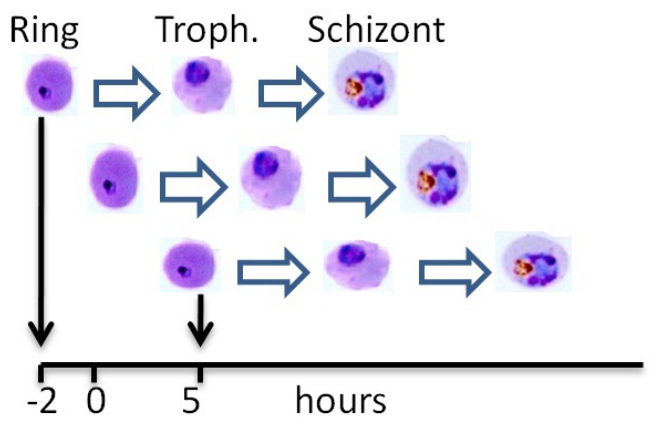

Figure 1. Diversity of infection time among iRBCs

\section{Analysis of decaying synchrony}

We assume that there are three main factors which drive the iRBCs out of synchronization in the experiment.

In the original experimental setup, late-stage Schizonts are synchronized by six sorbitol treatments on three generations [5], [10]. Prior to the first microarray time point, fresh RBCs are infected by Schizonts within two hours. After the two-hour-invasion, the concentration of RBCs is reduced from 14 percent to 3.3 percent to reduce the invasion of remaining RBCs. Our first concern is that the invasion of RBCs did not occur simultaneously in the two-hour-invasion. Furthermore, still can RBCs be infected as long as Schizonts remain after the two-hour-invasion, as shown in Fig. 1. In the experiment, considerable Schizonts are alive for five hours after the two-hour-invasion.

Let $N(t)$ be the total number of iRBCs at time $t$, and $n(t)$ be the derivative function of $N(t)$. In the perfectly synchronized case that all RBCs are simultaneously infected, $n(t)$ should have positive value only at one time point. In practical situation, however, $n(t)$ has a high value during the two-hour invasion, and it maintains positive for five hours after concentration is reduced to 3.3 percent. Actually the value of $n(t)$ can be monitored during the microarray experiment. As the original data set did not report this data, we assume that $n(t)$ is in directly proportional to the concentration of RBCs. Hence it can be approximated as a piecewise function:

$$
\frac{\mathrm{d} N(t)}{\mathrm{d} t}=n(t)= \begin{cases}14, & \text { if }-2 \leq t<0 \\ 3.3, & \text { if } 0 \leq t \leq 5 \\ 0, & \text { otherwise. }\end{cases}
$$

Let $f_{i}(s)$ be the expression level of protein $i$ from the perfectly synchronized iRBCs at their cell age $s$, where $s$ is the product of the hour of infection $(h)$ and growth rate $(r): s=h r$. For instance, one cell is infected at time $t_{1}$, its expression level on the protein $i$ at time $t_{2}$ is described as $f_{i}\left[\left(t_{2}-t_{1}\right) r\right]$. It is also observed in the experiment that iRBCs grow with different growth rates [5], [10]. For example, if a large amount of RBCs are simultaneously 


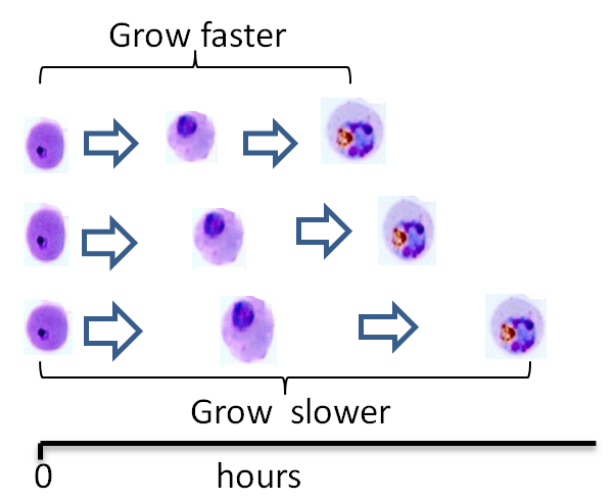

Figure 2. Diversity of growth rate among iRBCs

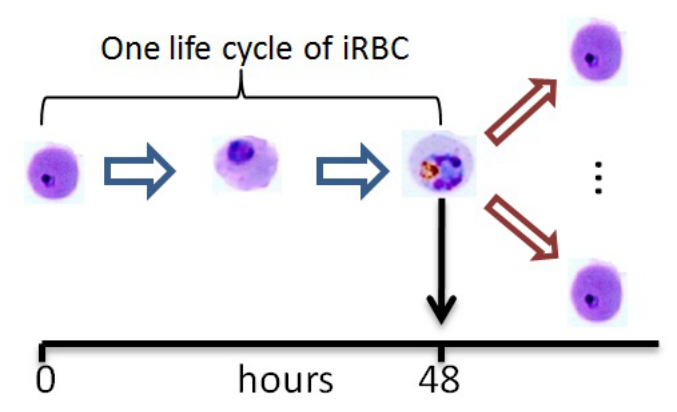

Figure 3. Newly infected RBCs

infected in the beginning, after several hours few iRBCs can reach the next stage faster than the majority of the iRBCs. This phenomenon give rise to our second concern that the diversity of growth rate gradually decays the synchrony, even if RBCs are well synchronized in the invasion period, as shown in Fig. 2. In this work, the random variable of growth rate is assumed to follow the Normal distribution: $r \sim N\left(1, \sigma^{2}\right)$. The value of $\sigma$ is adjusted to fit the experiment observations. The details will be discussed later.

Due to the diversity of growth rate, a few iRBCs can reach the late-stage of Schizont early. As a result, additional fresh RBCs will be infected by those fast-growing iRBCs, as shown in Fig. 3. This is also observed in the experiment [5], [10]. Let $N_{2}(t)$ be the number of RBCs which are infected in latter part of experiment at time $t . N_{1}(t)$ stands for the RBCs which reach the end of their life cycle at time $t . N_{2}(t)$ is approximated as a linear function on $N_{1}(t)$ :

$$
N_{2}(t)=a N_{1}(t)
$$

The invasion factor $a$ indicates the average number of fresh RBCs will be infected by one fast-growing iRBCs. Its value can be easily observed in the microarray experiment.

\section{Simulation of iRBCs population distribution}

From our above discussion, the distribution of cell age is gradually derived in the following. First the number of infected RBCs at time $t$ is described as:

$$
N(t)=\int_{-\infty}^{t} n\left(t^{\prime}\right) \mathrm{d} t^{\prime} .
$$

Let $p(h, s)$ be the probability that a RBC with $h$ hours of invasion reach the cell stage $s$. Therefore, the number of iRBCs which reach cell stage $s$ at time $t$ is given by:

$$
N(t, s)=\int_{-\infty}^{t} n(x) p(t-x, s) \mathrm{d} x .
$$

If we consider the RBCs which are infected by the fastgrowing iRBCs as well, (3) is updated as:

$$
N(t, s)=\int_{-\infty}^{t} n(x)[p(t-x, s)+a p(t-x, s+T)] \mathrm{d} x,
$$

where $T$ indicates the average life length of iRBCs.

As mentioned previously, $s=h r$ and $r \sim N\left(1, \sigma^{2}\right)$. Consequently, the cumulative distribution function of $p(h, s)$ is described by:

$$
\int_{-\infty}^{s} p(h, x) \mathrm{d} x=\Phi\left(\frac{s}{h}\right) .
$$

Then the equation for $p(h, s)$ can be derived as:

$$
p(h, s)=\frac{\mathrm{d} \Phi\left(\frac{s}{h}\right)}{\mathrm{d} s}=\frac{\phi\left(\frac{s}{h}\right)}{h} .
$$

Substituting (7) into (5), we have the expression of $N(t, s)$ :

$$
N(t, s)=\int_{-\infty}^{t} \frac{n(x)}{t-x}\left[\phi\left(\frac{s}{t-x}\right)+a \phi\left(\frac{s+T}{t-x}\right)\right] \mathrm{d} x,
$$

where

$$
\phi(x)=\frac{1}{\sqrt{2 \pi \sigma^{2}}} e^{-\frac{(x-1)^{2}}{2 \sigma^{2}}} .
$$

Determine the standard deviation of growth rate

The iRBCs population distributions described in (8) is subject to standard deviation of cell growth rate $\sigma$. To determine $\sigma$, its value is chosen such that iRBCs population distributions can ideally satisfy the observations in the experiment.

Over their 48-hour life cycle, iRBCs go through three life stages: Ring, Troph and Schizont. As shown in Fig. 4, the bounds of these three life stages for the perfectly synchronized iRBCs are derived from the percentage of iRBCs, which has been observed in the experiment [10]. The derived bounds are given as follow: 


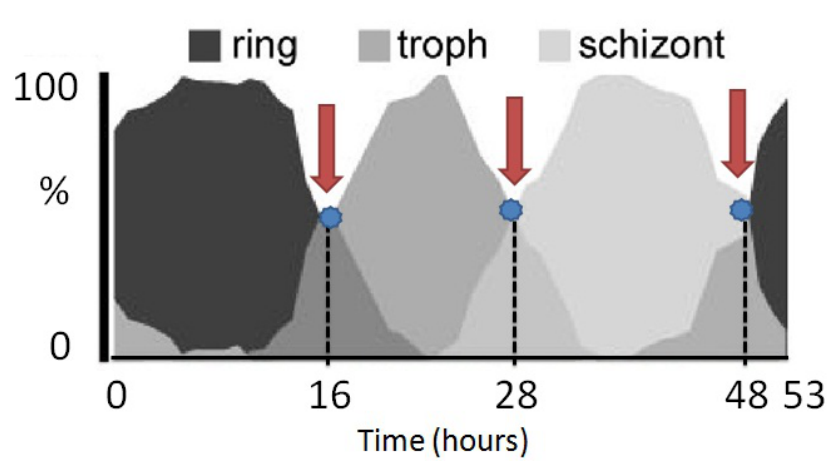

Figure 4. Percentage of Cells on Ring, Troph and Schizont observed in experiment

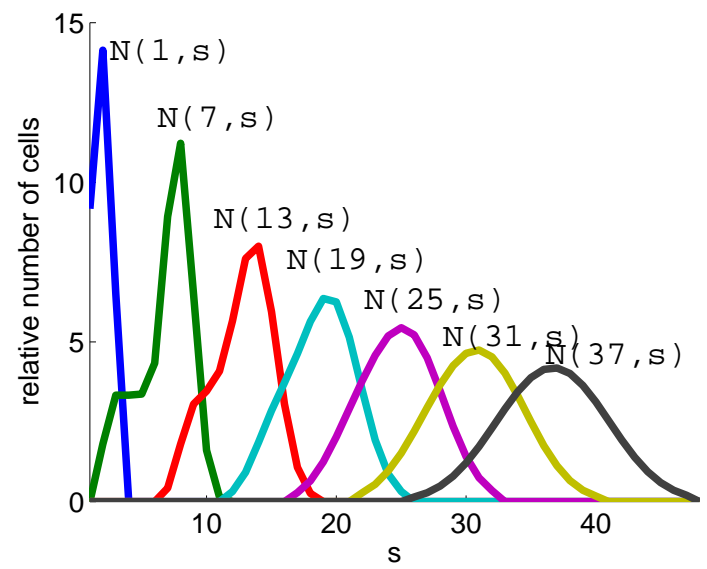

Figure 5. iRBCs population distributions change over time

RBC at cell stage $s= \begin{cases}\text { Ring, } & \text { if } 0 \leq s<16 \\ \text { Troph, } & \text { if } 16 \leq s<28 \\ \text { Schizont, } & \text { if } 28 \leq s \leq 48\end{cases}$

Based on above bounds, the percentage of iRBCs on Ring, Troph and Schizont can be calculated from iRBCs population distributions. Therefore, the value of $\sigma$ is tuned such that the iRBCs population distributions can produce the percentage of iRBCs coinciding with experimental observation. The iRBCs population distributions and calculated percentage of iRBCs are respectively given in Fig. 5 and Fig. 6.

\section{Modeling of gene expression level}

The gene expression levels obtained in the microarray experiment are the ensembles of individual iRBC. How $\mathrm{iRBCs}$ are accumulated together is modeled as a linear system. Let $f_{i}(s)$ be the expression level of individual iRBC on the protein $i$ when the iRBCs reach the cell stage $s$, and $e_{i}(t)$ indicates the observed expression level on the protein

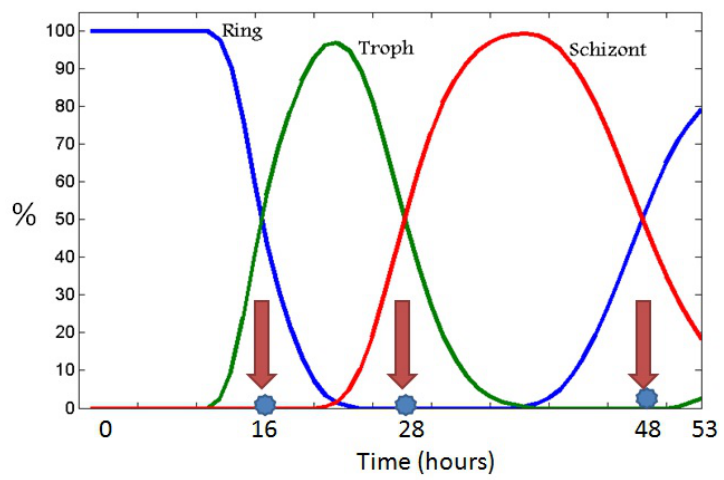

Figure 6. Percentage of iRBCs on Ring, Troph and Schizont calculated from iRBCs population distributions

$i$ at time $t$. As $N(t, s)$ denotes the number of iRBCs which reach cell stage $s$ at time $t, e_{i}(t)$ is described as:

$$
e_{i}(t)=\int N(t, s) f_{i}(s) \mathrm{d} s
$$

As the average life length of iRBCs is forty-eight hours, the continuous function $f_{i}(s)$ is approximated as a series of discrete points:

$$
\left\{f_{i}(1), f_{i}(2), \ldots, f_{i}(47), f_{i}(48)\right\} .
$$

The number of iRBCs at stage $s$ is also represented as discrete points:

$$
\{N(t, 1), N(t, 2), \ldots, N(t, 47), N(t, 48)\} .
$$

Therefore, the integral of $N(t, s)$ and $f_{i}(s)$ is approximated as:

$$
e_{i}(t)=\sum_{s=1}^{48} N(t, s) f_{i}(s) \triangle s .
$$

In the microarray experiment, the expression levels are measured from first time point to the fifty-third time point with one hour intervals. Therefore, a linear system can be derived based on (14):

$$
\underbrace{\left(\begin{array}{ccc}
N(1,1) & \ldots & N(1,48) \\
N(2,1) & \ldots & N(2,48) \\
\vdots & \ddots & \vdots \\
N(53,1) & \ldots & N(53,48)
\end{array}\right)}_{\mathbf{A}} \underbrace{\left(\begin{array}{c}
f_{i}(1) \\
f_{i}(2) \\
\vdots \\
f_{i}(48)
\end{array}\right)}_{\mathbf{x}}=\underbrace{\left(\begin{array}{c}
e_{i}(1) \\
e_{i}(2) \\
\vdots \\
e_{i}(53)
\end{array}\right)}_{\mathbf{b}}
$$

Notice that the above linear equations describes a discrete linear inverse problem. The $t$-th row of observation matrix $A$ denotes the iRBCs population distribution at the time point $t$. The unknown variable vector $x$ is the desired perfectlysynchronized expression curve on protein $i$. The constant 
vector, $b$, stands for the observed expression level of protein $i$.

\section{Reconstruction of synchronized expression level}

For each protein $i$, the observed expression level is modeled as the linear combination of the expression levels from individual iRBCs, as described in (15). As there are fiftythree equations with only forty-eight unknown variables, the linear system is overdetermined.

The objective function for the described inverse problem is derived in following. Firstly, a lower bound is assigned to the variables in $x$, because $x$ denotes the gene expression level whose value is valid only in positive range. Secondly, the idealized gene expression level is assumed to be a smooth curve over forty-eight time points. Consequently, the numerical solution is acquired by minimizing the objective function which consists of square error and the gradients of curve:

$$
x=\underset{x \geq 0}{\arg \min }[\underbrace{(A x-b)(A x-b)^{\top}}_{\text {square error }}+c \underbrace{\sum_{k=1}^{48}\left(x_{k}-x_{k+1}\right)^{2}}_{\text {gradients }}] .
$$

The problem described above is solved by the function fmincon in Matlab (MATLAB 7.9, The MathWorks Inc.)

\section{Prediction of protein kinases}

As we discussed above, high gene expression level is regarded as a sign that the function of corresponding protein is required. Let $\widehat{f}_{i}(t)$ be the normalized expression curve whose integral on one iRBC life length is equal to value one:

$$
\widehat{f}_{i}(t)=\frac{1}{\int_{0}^{48} f_{i}(x) \mathrm{d} x} f_{i}(t) \approx \frac{1}{\sum_{k=1}^{48} f_{i}(k) \triangle k} f_{i}(t),
$$

where $\mathrm{t}=1, \cdots, 48$.

The likelihood that the protein kinases $i$ is involved in regulating the stage transition can be estimated as:

$$
\int_{a}^{b} \widehat{f}_{i}(t) \mathrm{d} t \approx \sum_{t=a}^{b} \widehat{f}_{i}(t) \triangle t
$$

where $a$ and $b$ indicate the time period when the stage transition occurs.

According to the stage bounds described in (10), the stage transitions between Ring, Troph and Schizont are estimated to occur in the following three time-periods: from fifteen to seventeen, from twenty-seven to twenty-nine and from forty-seven to forty-eight returning to the period between one and two. Therefore protein kinase can be prioritized by its likelihood of involving in the desired stage transition.



Figure 7. Expression level of MAL7P1.144

\section{RESULTS AND DISCUSSION}

The synchronized gene expression levels are reconstructed for eighty-two protein kinases. The proteins kinases which may regulate the stage transitions are identified by the likelihood calculated from (18). As highlighted in the Table I and II, there are four protein kinases displaying high likelihood of regulating the transition from Schizont to Ring. There is only one protein kinase with relative high likelihood involving in the transition from Ring to Troph. None of the protein kinase has been found to be apparently involved in the transition from Troph to Schizont. Their synchronized expression levels are also plotted from Fig. 7 to 11 respectively.

As we mentioned earlier, it have been reported in recent studies that PFB0815w and PF13_0211 are involved in regulating the transition from Schizont to Ring [1], [2]. In our study, their likelihood of regulating the transition from Schizont to Ring are estimated as 0.387 and 0.391 respectively, ranking in the top four of all eighty-two protein kinases. This observation considerably enhances our confidence towards the accuracy of our predictions. We call for further experimental study to investigate the function of other protein kinases we have identified: PFB0665w and PF11_0267.

\section{CONCLUSIONS}

This paper presents a computational approach to predict the transcriptionally regulated protein kinases involved in controlling Plasmodium falciparum blood stage development. Firstly, we analyze the decaying synchrony among iRBCs in microarray experiment and proposed a method to reconstruct the synchronized gene expression level from observed microarray data. Secondly, the desired protein kinases are identified by the peaks in reconstructed gene expression level. By analyzing publicly available data set, a few protein kinases are predicted to be involved in the 
Table I

LIKELIHOOD OF BEING INVOLVED IN THE STAGE TRANSITIONS(PART 1)
Table II

LIKELIHOOD OF BEING INVOLVED IN THE STAGE TRANSITIONS(PART 2)

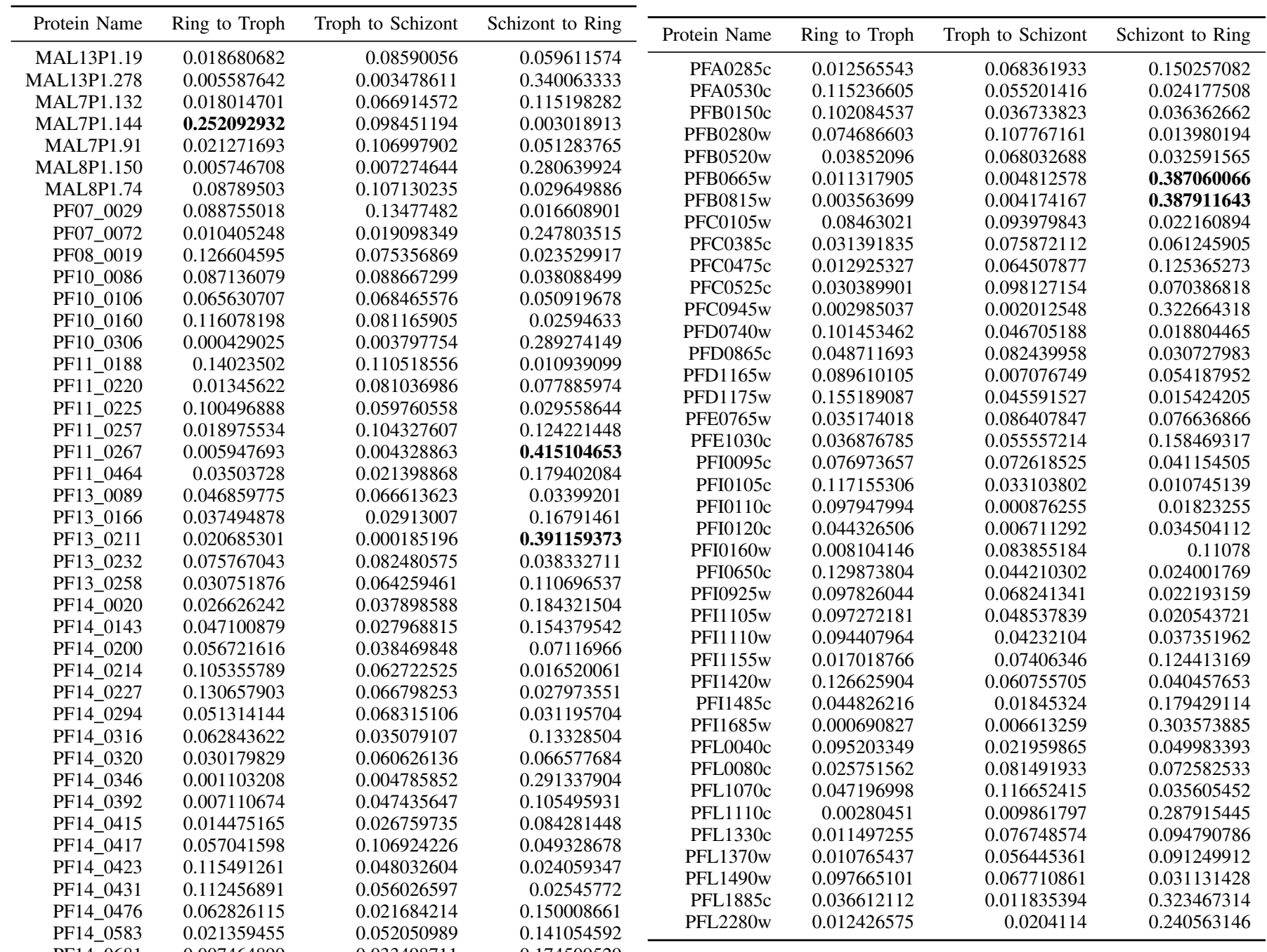

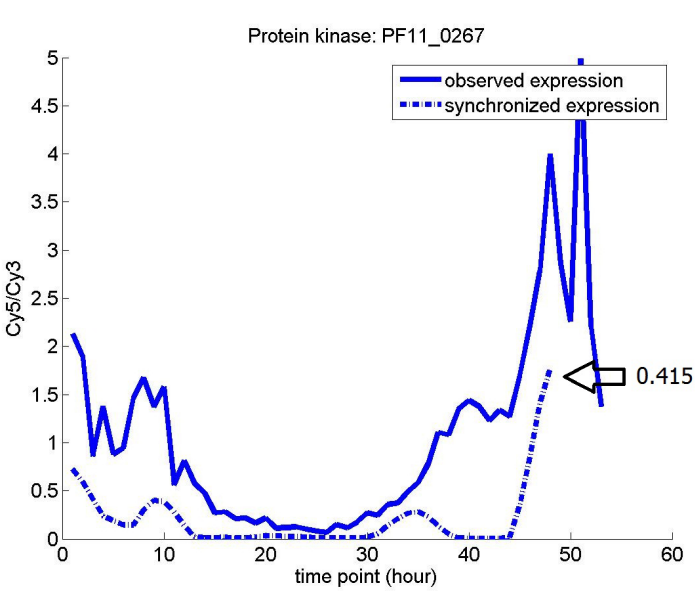

Figure 8. Expression level of PF11_0267

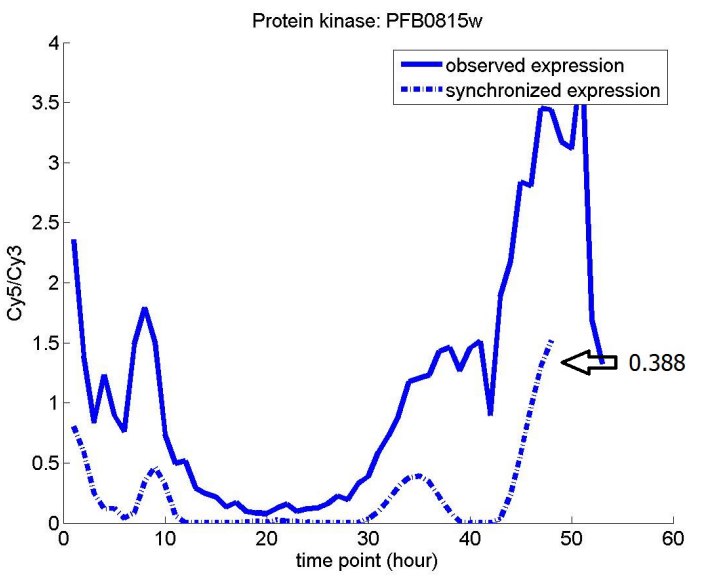

Figure 9. Expression level of PF13_0211 


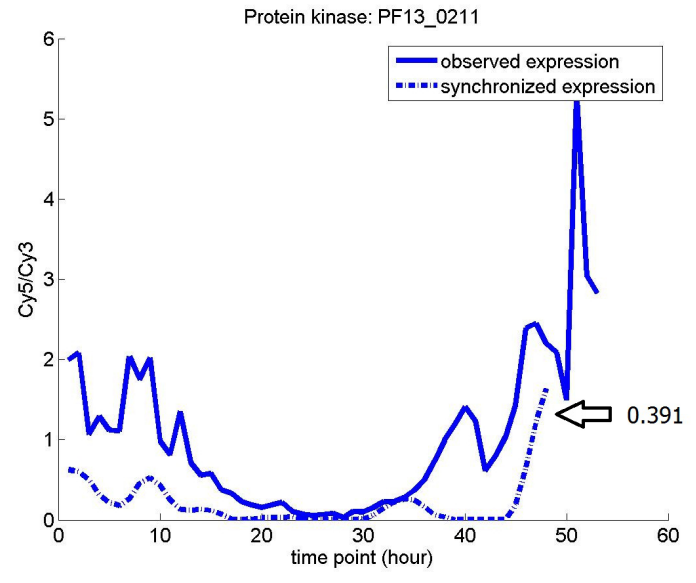

Figure 10. Expression level of PFB0815w

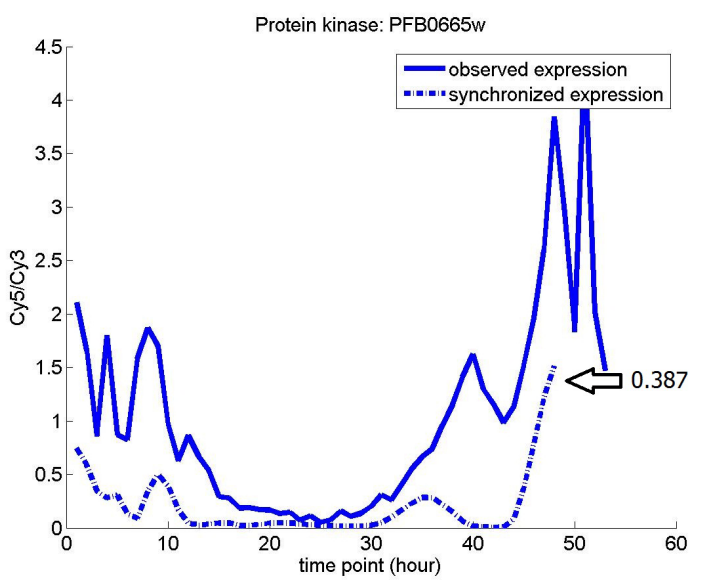

Figure 11. Expression level of PFB0665w

stage transition. Our prediction on two of these (PFB0815w and PF13_0211) have been validated in previous studies [1], [2]. Overall, these results suggest that further study on other protein kinases we have predicted may reveal new insights into $P$. falciparum blood stage development for instance PFB0665w and PF11 0267.

\section{REFERENCES}

[1] J. D. Dvorin, D. C. Martyn, S. D. Patel, J. S. Grimley, C. R. Collins, C. S. Hopp, A. T. Bright, S. Westenberger, E. Winzeler, M. J. Blackman, D. A. Baker, T. J. Wandless, and M. T. Duraisingh, "A Plant-Like Kinase in Plasmodium falciparum Regulates Parasite Egress from Erythrocytes," Science, vol. 328, no. 5980, pp. 910-912, May 2010. [Online]. Available: http://dx.doi.org/10.1126/science.1188191

[2] N. Kato, T. Sakata, G. Breton, K. G. Le Roch, A. Nagle, C. Andersen, B. Bursulaya, K. Henson, J. Johnson, K. A. Kumar, F. Marr, D. Mason, C. McNamara, D. Plouffe, V. Ramachandran, M. Spooner, T. Tuntland, Y. Zhou, E. C. Peters, A. Chatterjee, P. G. Schultz, G. E. Ward, N. Gray, J. Harper, and E. A. Winzeler, "Gene expression signatures and small-molecule compounds link a protein kinase to Plasmodium falciparum motility," Nature Chemical Biology, vol. 4, no. 6, pp. 347-356, May 2008. [Online]. Available: http://dx.doi.org/10.1038/nchembio.87

[3] M. C. Nunes, M. Okada, C. Scheidig-Benatar, B. M. Cooke, and A. Scherf, "Plasmodium falciparum FIKK Kinase Members Target Distinct Components of the Erythrocyte Membrane," PLOS ONE, vol. 5, no. 7, pp. e11747+, Jul. 2010. [Online]. Available: http://dx.doi.org/10.1371/journal. pone. 0011747

[4] W. H. Organization, World Malaria Report 2008, Geneva, Switzerland, 2008. [Online]. Available: http://www.who.int/ malaria/publications/atoz/9789241563697/en/index.html

[5] Z. Bozdech, M. Llinás, B. Lee, E. D. Wong, J. Zhu, and J. L. DeRisi, "The transcriptome of the intraerythrocytic developmental cycle of Plasmodium falciparum." PLoS biology, vol. 1, no. 1, pp. e5+, Oct. 2003. [Online]. Available: http://dx.doi.org/10.1371/journal.pbio.0000005

[6] K. G. Le Roch, Y. Zhou, P. L. Blair, M. Grainger, J. K. Moch, J. D. Haynes, P. De la Vega, A. A. Holder, S. Batalov, D. J. Carucci, and E. A. Winzeler, "Discovery of gene function by expression profiling of the malaria parasite life cycle." Science (New York, N.Y.), vol. 301, no. 5639, pp. 1503-1508, Sep. 2003. [Online]. Available: http://dx.doi.org/10.1126/science.1087025

[7] Anamika, N. Srinivasan, and A. Krupa, "A genomic perspective of protein kinases in Plasmodium falciparum." Proteins, vol. 58, no. 1, pp. 180-189, Jan. 2005. [Online]. Available: http://dx.doi.org/10.1002/prot.20278

[8] C. Doerig, O. Billker, D. Pratt, and J. Endicott, "Protein kinases as targets for antimalarial intervention: Kinomics, structure-based design, transmission-blockade, and targeting host cell enzymes." Biochimica et biophysica acta, vol. 1754, no. 1-2, pp. 132-150, Dec. 2005. [Online]. Available: http://dx.doi.org/10.1016/j.bbapap.2005.08.027

[9] K. G. Le Roch, J. R. Johnson, L. Florens, Y. Zhou, A. Santrosyan, M. Grainger, S. F. Yan, K. C. Williamson, A. A. Holder, D. J. Carucci, J. R. Yates, and E. A. Winzeler, "Global analysis of transcript and protein levels across the Plasmodium falciparum life cycle," Genome Research, vol. 14, no. 11, pp. 2308-2318, Nov. 2004. [Online]. Available: http://dx.doi.org/10.1101/gr.2523904

[10] M. Llinás, Z. Bozdech, E. D. Wong, A. T. Adai, and J. L. DeRisi, "Comparative whole genome transcriptome analysis of three Plasmodium falciparum strains." Nucleic acids research, vol. 34, no. 4, pp. 1166-1173, 2006. [Online]. Available: http://dx.doi.org/10.1093/nar/gkj517

[11] D. Siegal-Gaskins, J. N. Ash, and S. Crosson, "Model-Based Deconvolution of Cell Cycle Time-Series Data Reveals Gene Expression Details at High Resolution," PLoS Comput Biol, vol. 5, no. 8, pp. e1 $000460+$, Aug. 2009. [Online]. Available: http://dx.doi.org/10.1371/journal.pcbi.1000460 